\title{
A MIRROR FUSION DEVICE FOR ADVANCED SPACE PROPULSION
}

\author{
Terry Kammash and Myoung-Jae Lee \\ Department of Nuclear Engineering \\ The University of Michigan \\ Ann Arbor, MI 48109 \\ (313) 764-0205
}

\begin{abstract}
$\underline{\text { Abstract }}$
An open-ended fusion device with a magnetic mirror geometry is examined as a potential propulsion system that may be achievable with present day or near term technology. The plasma in such a device will be sufficiently dense to make the collision mean free path much smaller then the length of the system, thereby acquiring a confinement property which is significantly different from its terrestrial power-reactor counterpart. It is shown that such a system is capable of producing well over a hundred thousand seconds of specific impulse and tens of kilonewtons of thrust - a capability that would allow a massive rocket to make a round trip to Mars in about four months.
\end{abstract}

\section{INTRODUCTION}

The most critical requirement of a propulsion system that may be utilized in manned missions to the planets is the ability to make such journeys in a relatively short time so as to minimize the physical degradation, and exposure to galactic radiation, by the crew. Chemical, electric or even solid core nuclear thermal propulsion are incapable of meeting these requirements since the specific impulses, $I_{s p}$, they are known to generate generally do not exceed 1000 seconds. Although the open-cycle gas core nuclear rocket (GCR) is supposed to meet these stringent requirements, recent thermal hydraulic (Poston and Kammash 1994) and neutronic studies (Poston and Kammash 1994) have revealed a serious drop in its propulsive capability when material and hydrodynamic considerations are taken into account. With fusion fuel possessing a very high energy density, it appears that this approach to propulsion might prove to be the logical one to pursue in order to meet these objectives. Although there are many approaches to fusion, both magnetic and inertial, the fact remains that only those with simple geometry and relative ease of assembly make strong candidates. The device we propose in this paper is one such concept. It consists of a solenoid around which a current carrying conductor is wound to generate the simple mirror magnetic geometry illustrated in Figure 1. A hot plasma is maintained in steady state in this machine by injection of particles in the region of the homogeneous magnetic field to effectively balance the loss through the mirrors where the magnetic field is stronger than it is at the center. With $R_{0}$ denoting the vacuum field mirror ratio, it is important to impose some degree of asymmetry on this parameter in order to minimize escape through the mirror which will not be used as a nozzle in this propulsion system. In fact for a device with a large aspect ratio $\left(L \gg r_{p}\right)$ the magnetic geometry is effectively that of a "meridional" nozzle (Gerwin et al. 1990) which is azimuthally symmetric in that the fluid flow velocity is everywhere parallel to the magnetic field lines. In contrast to a conventional mirror fusion reactor where a typical fuel ion will traverse the device several times before it undergoes a scattering collision, the plasma in this propulsion device will be sufficiently dense that the collision mean free path, $\lambda$, will be significantly smaller than the length, $L$, and the plasma will behave much like a continuous medium - a fluid. Under these circumstances the escape of the plasma from the system is analogous to the flow of a gas into a vacuum from a vessel with a hole. The plasma "flux" across the mirror cross section, $A_{0}$, can be estimated as $A_{0} n v_{t h}$ where $n$ is the plasma density and $v_{t h}$ is the particle thermal velocity. If we divide the total number of particles in the system, $A_{c} n L$ ( $A_{c}$ is the cross sectional area of the plasma in the central region) by the flux we obtain the particle lifetime which can be expressed as

$$
\tau \sim \frac{A_{c} L}{A_{0} v_{t h}}=\frac{R L}{v_{t h}}
$$

where $R$ ( $=R_{0} / \sqrt{1-\beta}$ with $\beta$ to be defined later) is the mirror ratio seen by the plasma. A more careful analysis (Kammash et al. 1994) that takes into account the plasma potential that results from the rapid escape of the electrons shows that the correction to Equation (1) for most cases of interest is sufficiently small to be neglected. The energy with which the electrons and ions escape through the mirror is indeed affected by the presence of the electrostatic 

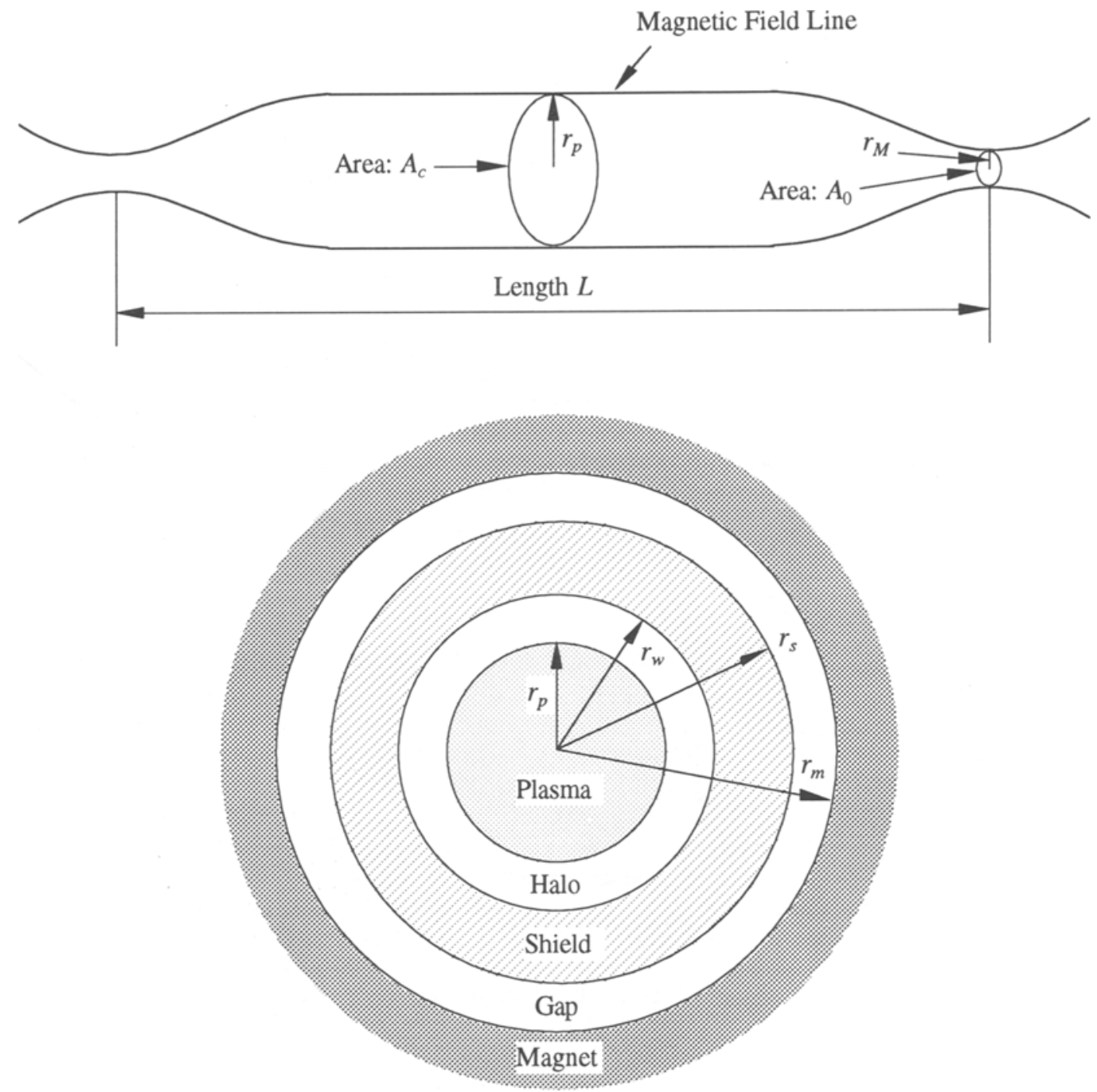

FIGURE 1. Schematic and Cross-sectional View of the Gasdynamic Fusion Propulsion System.

potential (which turns out to be equal to the electron temperature) and must be taken into account when computing the thrust generated by the machine. However in the interest of simplicity, we shall assume that Equation (1) is applicable to all the components of the plasma, and noting that the velocity distribution in a fluid is Maxwellian, it can readily be shown that the escape energy is given in terms of the temperature as

$$
E_{L}=2 T \text {. }
$$

In contrast with the classical mirror where $\lambda \gg L$, the lifetime in this device is a linear, rather than a logarithmic, function of $R$. Hence in a gasdynamic confinement system, increasing the mirror ratio to the highest value permitted by the technology provides a much greater effect than in a classical mirror. As will be seen shortly, devices with $R$ $\gg 1$ are particularly desirable as propulsion systems, and for such machines the estimate given by Equation (1) becomes valid when $\lambda / R<<L$ which is much less stringent than the condition $\lambda \ll L$. This implies that the critical parameter is not just the mean free path, $\lambda$, but an effective mean free path against scattering through an angle of the order of the "loss cone" angle $\theta \sim 1 / \sqrt{R}$. Another feature of the gasdynamic mirror is the high plasma density in 
the region just beyond the mirror where it is comparable to that at the center of the system. This feature makes it possible to suppress the "flute" instability which is known to plague the classical axisymmetric mirrors. Stability against this magnetohydrodynamic (MHD) mode, and against a microinstability associated with the loss cone noted above, provide an added dimension to the potential utility of this concept as propulsion device.

\section{BASIC EQUATIONS AND ANALYSIS}

A full assessment of the performance of this rocket requires solving the dynamic equations of all the species that make up the plasma. For a 50\%-50\% deuterium-tritium (DT) mixture these balance equations cover the ions, the electrons, and the alpha particles generated by the fusion reactions. The alpha particles are classified into two categories: the fast group represented by the energy range that begins with the birth energy $(3.5 \mathrm{MeV})$ and ends with the thermalization energy, and the thermal group which had thermalized on the background plasma and acquired a temperature not much different than that of the ions or the electrons. Rather than follow this detailed model that requires solving many coupled differential equations, we adopt a simplified model which allows us to evaluate, perhaps in a cursory fashion, the gasdynamic mirror as a propulsion device. We focus on the following two steady state equations that represent the mass and energy conservations:

$$
s-\frac{n}{\tau}-\frac{n^{2}}{2}\langle\sigma v\rangle=0
$$

and

$$
S E_{i n}+\frac{n^{2}}{4}\langle\sigma v\rangle E_{0}-\frac{n E_{L}}{\tau}-P_{b}-P_{s}=0 .
$$

In these equations $S$ is the rate of injection of fuel ions per unit volume, $\langle\sigma v\rangle$ the velocity-averaged fusion reaction cross section, $E_{\text {in }}$ the energy of the injected particle, $E_{0}$ the energy produced by a D-T fusion reaction (17.6 MeV), $P_{b}$ the bremsstrahlung radiation power, $P_{s}$ the synchrotron radiation power and $E_{L}$ the mean energy of an escaping ion given by Equation (2). The radiative power terms can be written as

$$
P_{b}=3.34 \times 10^{-15} n^{2} T^{1 / 2}=p_{0} n^{2} T^{1 / 2} \quad\left[\mathrm{keV} / \mathrm{cm}^{3}-\mathrm{sec}\right]
$$

and

$$
P_{s}=\frac{3.12 \times 10^{-16}}{\beta} n^{2} T^{2}\left[1+\frac{T}{204}\right]\left(1-R_{e}\right)=s_{0} n^{2} T^{2} \quad\left[\mathrm{keV} / \mathrm{cm}^{3}-\mathrm{sec}\right]
$$

where $R_{e}$ is the reflection coefficient of the walls surrounding the plasma, and $\beta$ is the ratio of the plasma pressure to the (vacuum) magnetic field pressure. For a hydromagnetically stable plasma, as we suggest it is in the gasdynamic mirror, the $\beta$ value can be chosen close to unity, i.e., $\beta \rightarrow 1$, which according to Equation (6) reduces the synchrotron radiation when compared to a lower $\beta$ device that generally characterizes the standard classical mirror. The above equation also reveals that for a highly reflecting wall most of this radiation stays in the plasma and does not go out to where it may constitute a hazard. In order to solve the conservation equations we need to determine the injection energy, $E_{i n}$, and for that we first draw on the standard definition of the gain factor " $Q$ ", i.e.,

$$
Q=\frac{n^{2}}{4}\langle\sigma v\rangle E_{0} / \frac{n E_{\text {in }}}{\tau}
$$

and noting that elimination of $S$ from Eqs. (3) and (4) yields

$$
L=\frac{\sqrt{8}\left(E_{i n}-2 T\right)}{n R \sqrt{\pi m}\left[p_{0}+s_{0} T^{\frac{3}{2}}-\frac{1}{4}\langle\sigma v\rangle\left(2 E_{i n}+E_{0}\right) T^{-\frac{1}{2}}\right]} .
$$


We see readily that

$$
E_{\text {in }}=-\frac{\xi}{4}+\sqrt{\left(\frac{\xi}{4}\right)^{2}+\frac{E_{0} E_{L}}{2 Q}}
$$

where

$$
\xi=E_{0}\left(\frac{1}{Q}+1\right)-\frac{4\left(P_{b}+P_{s}\right)}{n^{2}\langle\sigma v\rangle} .
$$

It is interesting to note that if the radiation terms are ignored the injection energy assumes the simple form:

$$
E_{\text {in }} \approx \frac{E_{L}}{Q+1}=\frac{2 T}{Q+1}
$$

which clearly indicates that a lower injection energy will be needed if the reaction is to produce power in excess of the amount required to sustain it.

\section{RESULTS AND DISCUSSION}

It is clear that from Equation (8) that the rocket length is inversely proportional to the plasma density and the plasma mirror ratio. These results are shown vividly in Figure 2 where we note that a rocket length of about $32 \mathrm{~m}$ obtains when a plasma density of $10^{16} \mathrm{~cm}^{-3}$ and a mirror ratio of 50 are used. The same figure reveals that for a fixed $R$, a one order of magnitude increase in the plasma density results in an equal drop in the rocket length. We observe also that a plasma temperature of about $15 \mathrm{keV}$ appears to be an optimum for the operation of this gasdynamic mirror fusion rocket, and in contrast to the terrestrial power reactors, smaller gain factors, i.e., $Q \geq 1$ are indeed more desirable as depicted in Figure 3.

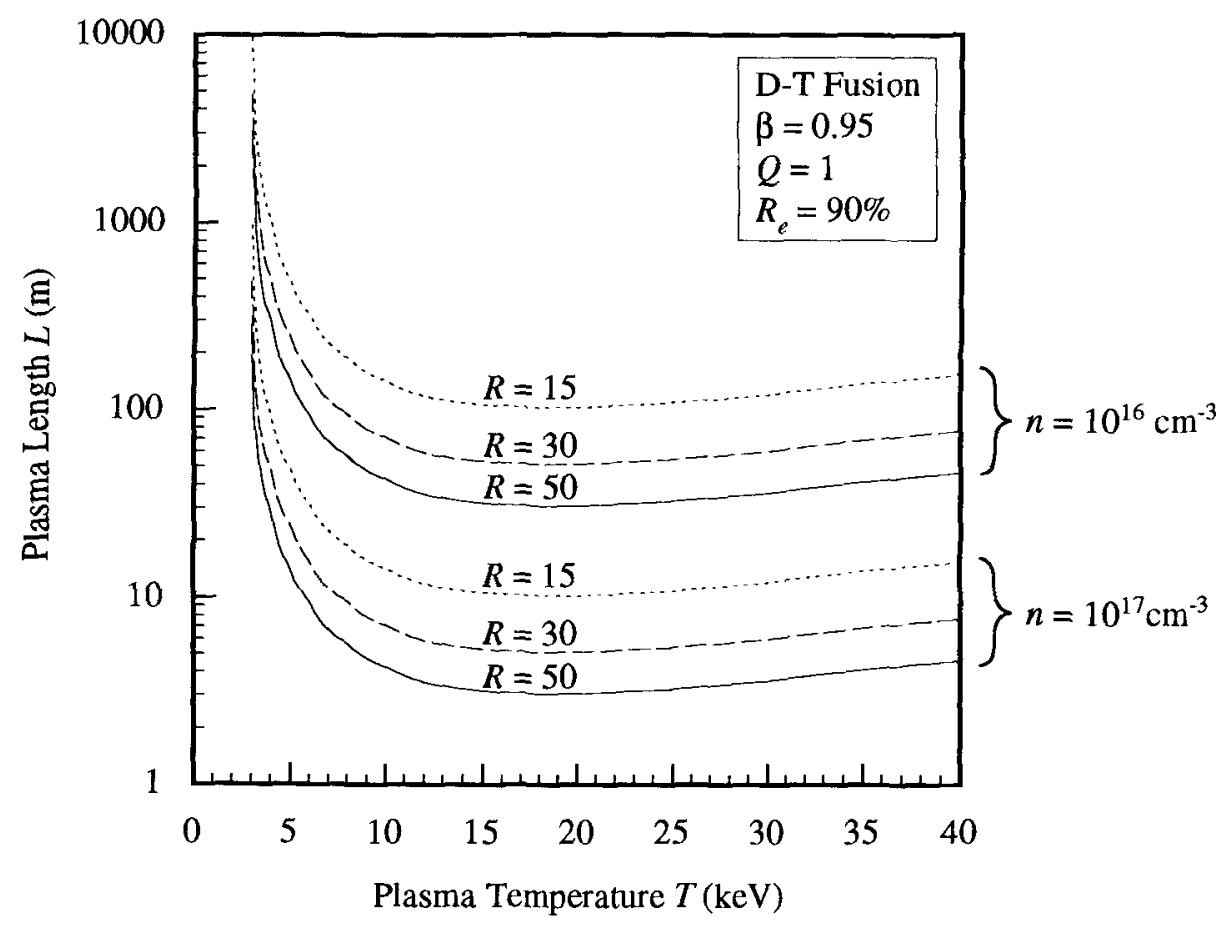

FIGURE 2. The Effect of Mirror Ratio on Plasma (Rocket) Length. 


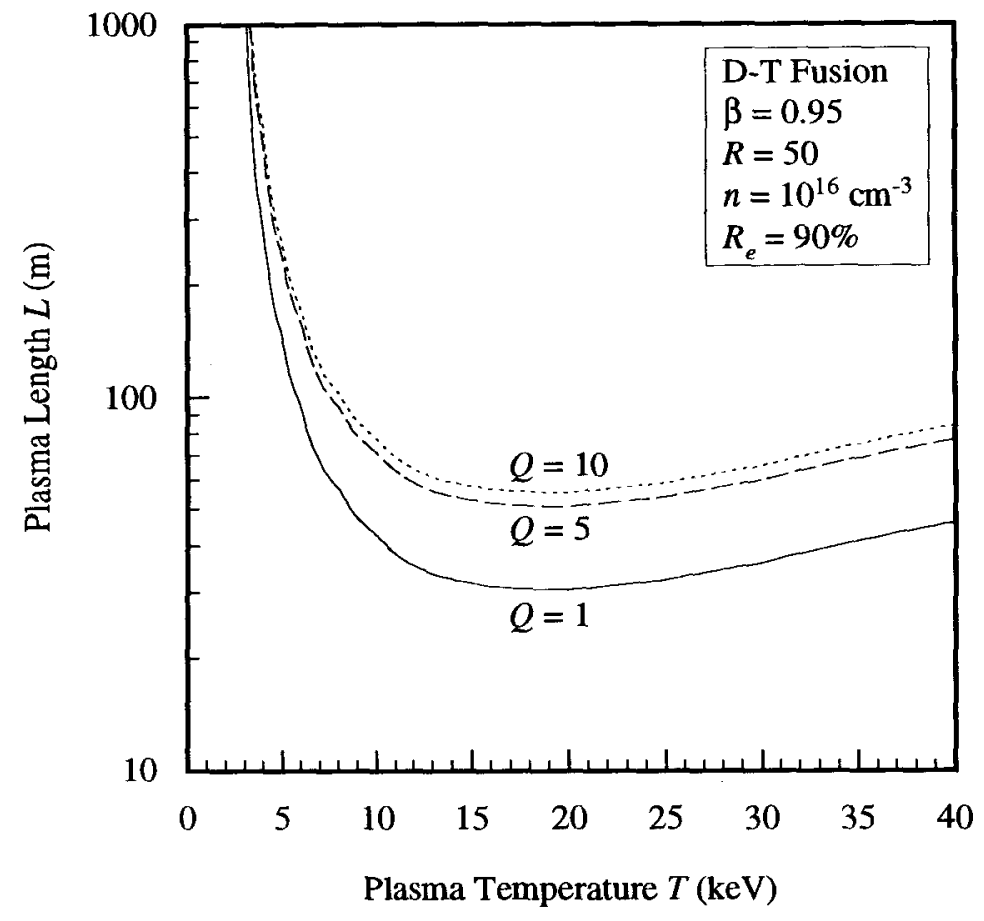

FIGURE 3. The Role of Reactor Gain Factor in Plasma (Rocket) Size.

\section{SUMMARY AND CONCLUSIONS}

We have proposed in this paper a gasdynamic mirror fusion reactor which could readily by utilized for rocket propulsion. It requires a high density plasma $\geq 10^{16} \mathrm{~cm}^{-3}$ in order for the confinement time to scale linearly with the mirror ratio, and the length of the system, i.e., for the plasma to behave like a fluid. Under these circumstances the plasma can achieve, through the fusion reactions, a temperature that can translate into a specific impulse of well above a hundred thousand seconds and a thrust of several tens of kilonewtons. The vacuum magnetic field required to confine the plasma in the center of the device is about 11 tesla which is not much larger than magnetic fields in use in some current experimental fusion devices.

\section{Acknowledgments}

The work was carried out at the Nuclear Engineering Department of The University of Michigan.

\section{References}

Gerwin, R. A., G. J. Marklin, A. G. Sgro and A. H. Glasser (1990) "Characterization of Plasma Flow Through Magnetic Nozzles," Astronautics Laboratory Report AL-TR-89-092.

Kammash, T., M. J. Lee and D. L. Galbraith (1994) "A High-Performance Fusion Rocket for Manned Space Missions," 30th AIAA/ASME/SAE/ASEE Joint Propulsion Conference, June 27-29, 1994/ Indianapolis, IN; Paper AIAA-94-3266. 
Poston, D. I. and T. Kammash (1994) "A Comprehensive Thermal-Hydraulic Model of an Open-Cycle Gas Core Nuclear Rocket," in Proc. Eleventh Symposium on Space Nuclear Power Systems, M. S. El-Genk and M. D. Hoover, eds., American Institute of Physics, New York, AIP Conference Proceedings, No. 301, 1: 473-479.

Poston, D. I. and T. Kammash (1994) "Hydrodynamic Fuel Containment in an Open-Cycle Gas Core Nuclear Rocket," in Proc. Eleventh Symposium on Space Nuclear Power Systems, M. S. El-Genk and M. D. Hoover, eds., American Institute of Physics, New York, AIP Conference Proceedings, No. 301, 3: 1415-1420. 